\title{
MAXWELL E GLI ANELLI DI SATURNO
}

\author{
Nota del m.e. SERGIO BITTANTI (*)
}

(Adunanza del 2 ottobre 2014)

SunTO. - Ancora studente all'Università di Cambridge, James Clerk Maxwell si cimentò nello studio degli anelli di Saturno, al fine di scoprirne la loro reale costituzione. Lo studio non fu basato su osservazioni astronomiche, ma su un approccio di pura modellistica matematica. Mediante la legge di gravitazione universale, Maxwell costruì vari modelli esplicativi, ciascuno dedotto in conformità con una determinata ipotesi di lavoro. Ad esempio, un modello fu costruito nell'ipotesi che l'anello fosse un'unica corona rigida, un altro era invece basato sull'ipotesi che si trattasse di una corona gassosa, e così via. Una volta costruiti i modelli, si passava alla fase di validazione effettuata per confronto con l'evidenza dei fatti. I modelli con caratteristiche contradditorie rispetto alla realtà dovevano essere scartati, e, con loro, le ipotesi su cui si basavano. Come strumento base per la validazione, Maxwell si avvalse della nozione di stabilità, partendo dall'assunzione che, essendo gli anelli in tale configurazione da millenni, il modello ottenuto dovesse essere necessariamente stabile; pertanto, i modelli instabili andavano rigettati, insieme con le sottostanti ipotesi. L'approccio, che anticipa le attuali tecniche di identificazione dei modelli, è sintetizzato magistralmente dall'autore con queste parole: ... by rejecting every bypothesis which leads to conclusions at variance with the facts, we may learn more on the nature of these distant bodies than the telescope can yet ascertain. Fu così che Maxwell concluse che gli anelli dovevano essere costituiti da molti elementi rigidi disconnessi tra loro, in rotazione intorno al pianeta, ciò che, a distanza di secoli, è stato confermato dalle odierne missioni spaziali.

$* * *$

ABSTRACT. - While still a student at Cambridge University, James Clerk Maxwell attention was attracted by Saturn Rings. To discover how these celestial bodies are composed, he constructed various mathematical models; each model was derived under dif-

(*) Dipartimento di Elettronica, Informazione e Bioingegneria del Politecnico di Milano, Milano, Italy.

E-mail: sergio.bittanti@polimi.it 
ferent assumptions, such as: the rings constitute a unique rigid cap, or the rings are made by a gas nebula, and so on. The adopted rationale was to construct the models and then validate them by comparing their characteristics with "the facts", as Maxwell wrote, namely with the observation of reality. For the validation, Maxwell resorted to the notion of stability. Indeed, being the rings operating since millennia, it was argued that the stability property had to be satisfied. In the opposite, the model had to be rejected, together with the underlying assumption. This approach, which anticipates the current identification techniques, is summarized by the author with this masterful statement: ... by rejecting every bypothesis which leads to conclusions at variance with the facts, we may learn more on the nature of these distant bodies than the telescope can yet ascertain. With his approach, Maxwell came to the conclusion that the rings are constituted by various rigid parts disconnected each other rotating around the planet, a fact which has been confirmed by space missions of our days.

\section{LA SCOPERTA Di NeTtUNO E IL PREMio AdAMS}

Alla metà dell'ottocento, John Adams, docente all'Università di Cambridge, si era concentrato sullo studio delle traiettorie dell'ultimo pianeta allora conosciuto, Urano. Adams giunse alla conclusione che tali traiettorie non potevano che essere spiegate con l'esistenza di un altro corpo celeste, un pianeta esterno. La medesima conclusione fu tratta, in modo indipendente, da Urbain Le Verrier, che lavorava a Parigi. Grazie ai loro calcoli, fu possibile stimare la posizione del "nuovo" corpo celeste, corpo che fu osservato sperimentalmente da Johan G. Galle, con il telescopio dell'osservatorio di Berlino. Era il 23 settembre 1846. Il sistema solare si arricchiva così di un nuovo pianeta, cui fu attribuito il nome di Nettuno.

Per dare lustro a questa scoperta, nel 1848 l'Università di Cambridge istituì un riconoscimento denominato Premio Adams, il cui bando recita:

The University has accepted a fund raised by several members of St John's College, for the purpose of founding a Prize to be called the ADAMS PRIZE, for the best essay on some subject of Pure Mathematics, Astronomy, or other branch of Natural Philosophy.

Il premio era biennale e vi poteva partecipare chi era stato ammesso, in qualunque epoca, a quell'Università, dunque a tutti gli alunni, presenti e passati.

In questa nota, focalizzeremo l'attenzione su due Premi Adams, quello del 1855 e quello del 1876. Iniziamo con il primo, del secondo parleremo più avanti. 
Il bando del 1855 diceva quanto segue:

The Examiners give Notice, that the following is the subject for the prize to be adjudged in 1857: - "The Motions of Saturn's Rings".

Questo tema si collocava nell'ambito degli studi sul sistema solare compiuti nel corso del settecento e dell'ottocento, [1].

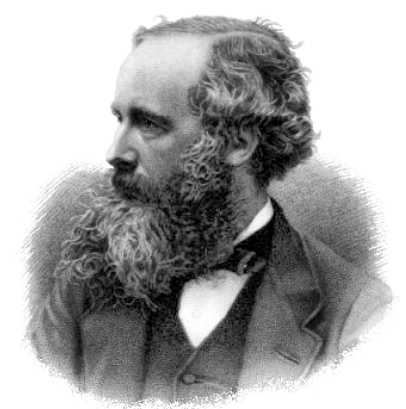

Fig. 1 - Ritratto di James Clerk Maxwell.

Il premio andò a un giovane studente, James Clerk Maxwell (Fig. 1), originario di Edimburgo, dove era nato il 13 giugno 1831, per il saggio "The stability of Saturn's rings", la cui copertina è riprodotta in Fig. 2. Si osservi che il titolo del saggio non coincide esattamente con il tema del bando.

ON THE

\section{STABILITY OF THE MOTION}

or

SAT URN'S R I NGS.

A N E S S A Y,

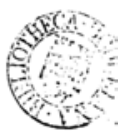

WHICH OBTAINED THE ADAMB PRIZE FOR THE YBAR 1866, IN THE UNIVERSITY OF CAMBRIDGE.

Fig. 2 - Frontespizio del saggio di Maxwell sugli anelli di Saturno. 


\section{PRIMI STUDI SUL SISTEMA SOLARE}

Il libro di Newton "Philosophiae Naturalis Principia Mathematica", pubblicato nel 1687, offrì al mondo i metodi per la comprensione delle leggi che governano i fenomeni celesti, da sempre oggetto d'investigazione da parte degli esseri umani.

Ebbe così avvio un fervore di studi sul sistema solare. Una delle domande che ci si poneva era quale effetto avrebbe potuto avere una perturbazione dovuta all'intrusione di un corpo esterno, come una cometa o un asteroide. ${ }^{1}$ La presenza di tale corpo avrebbe potuto avere effetti diversi sulle traiettorie dei pianeti, come a) una deformazione temporanea, al termine della quale i pianeti sarebbero poi tornati nelle loro usuali traiettorie, b) una deformazione permanente, c) la deflagrazione del sistema solare. Si trattava cioè di studiare la stabilità del sistema solare. Tra i paladini di questi studi di astronomia matematica si annoverano grandi scienziati, come Joseph-Louis Lagrange (17361813), Pierre Simon Laplace (1749-1827), Simon-Denis Poisson (17811840) e Carl Jacobi (1804-1851). In particolare, Laplace diede due fondamentali contributi, il saggio Exposition du système du monde (1796), e la monumentale Mécanique Céleste, un'opera che si compone di cinque volumi, il primo dei quali pubblicato nel 1799. Di Laplace, vogliamo anche ricordare il celebre motto: poco è ciò che conosco, immenso ciò che ignoro. Lo studio del sistema solare appassionava talmente l'opinione pubblica che, nel 1887, re Oscar di Svezia istituì un premioº per gli

1 Eventi di questo genere non sono così remoti. Ad esempio, nel 1994, la cometa Shoemaker-Levy si schiantò su Giove. Di recente, il 19 ottobre 2014, la cometa Siding Spring ha sfiorato Marte, passando a 140.000 chilometri dal pianeta rosso (vale a dire a un terzo della distanza Terra-Luna), con pesanti conseguenze sulla sua atmosfera. Anche la Terra fu minacciata da un simile evento, quando la cometa Lexel transitò a soli 2 milioni di kilometri da noi. Era il 1770.

2 Re Oscar istituì il premio per celebrare il suo sessantesimo genetliaco. Per la medesima occasione, l'autore di questa nota ebbe invece l'idea di proporre un numero speciale di European Journal of Control, rivista di cui fu Editor-in-Chief dal 2003 al 2013 , interamente dedicato agli albori della scienza del controllo. Il numero, curato con Michel Gevers, docente all'Università di Lovanio, raccoglie le testimonianze di emeriti studiosi di varie nazionalità: Karl Åström (per gli sviluppi in Svezia), Roger Brokett (US), Han-Fu Chen e Daizhan Cheng (Cina), A.J. Fossard (Francia), Guido Guardabassi (Italia, si veda il riferimento [9]), Alexander Kurzhanski (Russia), David Mayne (UK) e Jan Willems (Olanda). 
studi sul sistema dei tre corpi (ad esempio, Sole, Terra, Luna). Si trattava di determinare le traiettorie di tale sistema, note le condizioni iniziali. Il premio (si potrebbe dire il "premio Oscar") andò a Henri Poincaré (1854-1912), che mostrò l'impossibilità di esprimere la soluzione in forma esplicita, provando anche che due soluzioni associate a condizioni iniziali poco diverse potevano divergere tra loro a lungo andare.

\section{NASCITA DEL CONCETTO DI SISTEMA DINAMICO}

Poiché la forza determina l'accelerazione, che è la variazione della velocità, e, a sua volta, la velocità è, la variazione della posizione, la celebre legge di gravitazione universale si traduce matematicamente in un legame dinamico tra forza e posizione, ciò che oggi chiamiamo equazione differenziale. Nel mondo scientifico di allora, questo genere di modellizzazione era un'innovazione di grande portata. In precedenza, infatti, l'attenzione degli studiosi era sostanzialmente focalizzata su descrizioni di tipo algebrico.

Per maggiore chiarezza, soffermiamoci su questi due termini, relazione algebrica e relazione dinamica, nell'ipotesi semplificativa che il sistema che si stia analizzando sia caratterizzato da due sole variabili, diciamo $u$ e $y$, dipendenti dal tempo $t: u(t)$ e $y(t)$. Può essere utile pensare a $u(t)$ come la variabile causa e a $y(t)$ come la variabile effetto. Una relazione algebrica tra due variabili è una relazione che lega i valori istantanei delle variabili. In tal modo, $y(t)$ è perfettamente determinabile quando si conosce $u(t)$, il valore assunto dalla variabile $u$ al tempo $t$. Ad esempio, la tensione $y(t)$ ai capi di un filo conduttore (un resistore) è proporzionale alla corrente $u(t)$, come asserisce la celebre legge di Ohm. Vi sono però dei casi in cui non è possibile stabilire il valore di $y(t)$ noto che sia solamente $u(t)$. Consideriamo per esempio l'andamento $y(t)$ della popolazione in Cina. Nel periodo che va dal 1960 al 2010, $y(t)$ è crescente, passando da circa 0.6 a circa 1.5 miliardi di abitanti. Al contrario, il tasso di fertilità, vale a dire il numero medio di figli per donna, è decrescente, da circa 6 a circa 2 . Se consideriamo il tasso come "variabile causa", $u(t)$, sembrerebbe vi sia una contraddizione dato che, nonostante che la progressiva diminuzione di $u(t)$, la popolazione $y(t)$ è in continua crescita. In realtà, il paradosso è solo apparente; infatti, è a tutti chiaro che il valore attuale della popolazione dipende dall'andamento del tasso in tutta la sua storia passata. Presumibilmente, l'effetto della riduzione della fertilità si 
manifesterà tra qualche anno, anzi tra qualche decennio. Pertanto, il legame $u(t)-y(t)$ è, in questo caso, dinamico.

\subsection{Osservazione (sui modelli)}

La legge di gravitazione esplicita la forza di attrazione tra due corpi. Per come è enunciata normalmente, è sottinteso che si tratta di una forza che si "esercita a distanza", in modo istantaneo. Già Laplace, nel corso dei suoi studi sul moto della luna, aveva dubitato che questo fosse corretto; certe anomalie, infatti, non avrebbero potuto trovare spiegazione se la forza di gravità si fosse davvero trasmessa a velocità infinita. Per avere un completo chiarimento su questa fondamentale questione, bisognava però attendere i primi decenni del novecento, quando, per merito di Henry Poincaré e Albert Einstein, fu sviluppata la teoria della relatività. Divenne allora chiaro che la forza di gravità non si propaga da un corpo all'altro istantaneamente, ma a velocità finita, precisamente alla velocità della luce. Così, se in questo momento il sole cessasse la sua attività, la forza di gravitazione cui è soggetta la Terra continuerebbe a manifestarsi per altri otto minuti, il tempo impiegato dalla luce per giungere a noi. Naturalmente, questa osservazione, seppur di fondamentale importanza, non inficia la portata della legge di Newton, che, per i sistemi in cui i corpi si muovono a velocità lontane da quella della luce, ha consentito di raggiungere risultati formidabili per l'umanità. Del resto, com'era solito dire lo statistico George Box (1919-2013), tutti i modelli sono sbagliati, ma qualcuno è utile.

\section{Albori DEL CONCETTO DI STABILITÀ}

Come accennato in precedenza, gli studi sulla stabilità del sistema solare erano volti a stabilire l'effetto di una perturbazione prodotta da una cometa o da altro corpo celeste in transito nel sistema. Si tratta di un interessantissimo problema, che ha comprensibilmente suscitato l'interesse non solo degli studiosi ma anche del grande pubblico. A ben riflettere, però, il concetto di stabilità ha valenza generale, e può essere riferito a un qualunque sistema dinamico. In particolare, questa nozione ha un ruolo cruciale nei sistemi di controllo. Per il buon funzionamento di tali sistemi, l'effetto d'imprevedibili perturbazioni (disturbi) deve essere neutralizzato, e questo può accadere solo se il sistema è stabile. 
L'affermazione del concetto di stabilità per lo studio di ogni sistema dinamico ebbe luogo nella seconda metà dell'ottocento. Il merito della definizione generale, universalmente accettata ai nostri giorni, va ad Alexander M. Lyapunov (1857-1918), che l'enunciò per la prima volta nella sua tesi di dottorato, discussa all'Università di San Pietroburgo nel 1884.

Tuttavia, come vedremo a breve, la nozione di stabilità era già apparsa ed utilizzata in letteratura alcuni decenni prima. In particolare, tale nozione svolse un ruolo fondamentale già nei lavori di Maxwell.

\section{Il SAgGio di MAXwell SUgLi ANELLI Di SATURNO}

Veniamo ora al saggio sugli anelli di Saturno. Analizzeremo dapprima la logica di studio adottata. Passeremo quindi a descrivere l'organizzazione del saggio stesso

\subsection{La logica di studio adottata nel saggio anticipa le moderne tecniche d'identificazione dei modelli}

Nel suo saggio, Maxwell procede facendo diverse congetture sulla costituzione degli anelli e ricavando il corrispondente modello matematico. Il modello ottenuto viene quindi validato confrontando le sue caratteristiche salienti con "i fatti", vale a dire la realtà conosciuta. Se il modello è contradditorio rispetto al reale, l'ipotesi di partenza viene rigettata. Se non è contradditorio, allora la congettura non è falsificata, e potrebbe essere corretta. Nel saggio, vi è uno splendido passaggio in cui Maxwell sintetizza magistralmente questo suo modus operandi:

... by rejecting every hypothesis which leads to conclusions at variance with the facts, we may learn more on the nature of these distant bodies than the telescope can yet ascertain.

Notiamo che questa linea di pensiero è tipica di quella disciplina che chiamiamo oggi identificazione dei modelli e analisi dei dati, il cui obiettivo è di ricavare una descrizione matematica valida di un dato sistema da dati sperimentali, a scopo conoscitivo, previsionale o di controllo. A tal fine si postula inizialmente una famiglia di modelli, all'interno della quale si sceglie il "modello migliore", di norma inteso come quello che fornisce pre- 
dizioni aderenti il più possibile alle osservazioni sperimentali. Si valida poi il risultato ottenuto: se il modello non è soddisfacente, ad esempio perché qualche sua caratteristica non rispetta l'evidenza sperimentatale, si passa ad altra famiglia di modelli e si ripete il procedimento ex novo [2].

Ed ecco l'idea vincente di Maxwell: la validazione di un dato modello viene fatta ricorrendo alla nozione di stabilità: poiché' gli anelli sono in essere da tempo immemorabile, un modello valido deve essere stabile. In caso opposto, il modello va rigettato, e con esso l'ipotesi su cui esso si basa.

\subsection{Organizzazione del saggio}

Il saggio sugli anelli di Saturno si compone di 68 pagine seguite da una Appendice e da una tavola finale con le figure, 13 in tutto. I riferimenti bibliografici sono citati di volta in volta all'interno del testo.

Il lavoro è organizzato in due parti. La prima (pagine 6-17) ha come titolo On the motion of a rigid body of any form about a sphere. Qui il modello è ricavato nell'ipotesi che gli anelli siano costituiti da un unico blocco rigido. Come evidenzia Maxwell stesso nell'introduzione generale (pagine 1-5), tale ipotesi era già stata fatta nella Mécanique Céleste di Pierre Simon Laplace, per l'esattezza nel Capitolo sesto del Terzo volume e nel Capitolo terzo del Quinto volume. In merito, Maxwell scrive che

... (Laplace) proves most distinctly that a solid uniform ring cannot revolve around a central body in a permanent manner, for the slightest displacement of the centre of the ring from the centre of the planet would originate a motion which would never be checked, and would inevitably precipitate the ring upon the planet... We may draw the conclusion more formally as follows: If the rings were solid and uniform, their motion would be unstable, and they would be destroyed. But they are not destroyed, and their motion is stable; therefore they are either not uniform or not solid.

Nella seconda parte del saggio, intitolata On the motion of a ring, the parts of which are not rigidly connected, si studia il caso di più elementi non rigidamente connessi, come se l'anello fosse costituito da vari satelliti indipendenti. Naturalmente, la modellistica è più complessa poiché occorre considerare, oltre alla forza di attrazione esercitata da Saturno e alla forza centrifuga, anche l'effetto mutuo di un satellite sugli altri. Lo studio, viene condotto dietro alcune ipotesi semplificative: a) la traiettoria di ciascun satellite è circolare; b) la velocità di rotazione è uniforme; c) i 
vari satelliti dell'anello sono tutti identici tra loro d) la dimensione trasversale è trascurabile. Il numero dei satelliti è indicato con la lettera greca $\mu$, mentre $\mathrm{R}$ ed $\mathrm{S}$ indicano rispettivamente la massa dell'anello e la massa di Saturno. La condizione ricavata da Maxwell affinché il moto sia stabile è che la massa di Saturno sia sufficientemente elevata in rapporto a quella dell'anello: $\mathrm{S}>0.4352 \mu^{2} R$. Come osserva Maxwell stesso, se il numero di satelliti fosse così elevato da violare questa condizione, il moto sarebbe instabile, e si innescherebbero oscillazioni distruttive che porterebbero i satelliti a collidere tra loro e quindi - presumibilmente - ad unificarsi in un unico corpo, riportando il numero dei satelliti all'interno del massimo che Saturno può sostenere (o, come dice l'autore, keep in discipline). Quando al contrario la diseguaglianza è verificata, i satelliti non solo sono in grado di permanere ad una distanza appropriata dal centro di gravità di Saturno, ma, data la preponderante massa del pianeta, possono evitare di interferire l'un l'altro.

Nel corso della trattazione, i simboli impiegati, in numero non piccolo, vengono via via introdotti in modo discorsivo, ciò che rende la lettura un po' difficoltosa. Maxwell ha però il merito di terminare lo studio con un'utile ricapitolazione della prima e della seconda parte, facendo una sintesi efficace dei casi considerati e dei risultati ottenuti.

\subsection{Osservazione (sulle missioni spaziali per l'esplorazione di Saturno)}

Gli studi di Maxwell sulla costituzione degli anelli hanno trovato conferma nel corso di alcune missioni spaziali. Si tratta principalmente di Pioneer 11, Voyager 1, Voyager 2 e Cassini-Huygens, le cui sonde sono transitate nei pressi del grande pianeta nel 1979, 1980, 1981 e 1997, rispettivamente. Per un resoconto di queste missioni si rimanda a [3] e [4].

\section{Contributo di Maxwell allo STUdio DeI Sistemi DI CONTROLLO}

Ci soffermiamo ora su un altro importante contributo di Maxwell, in cui si usano alcuni concetti vicini a quelli già impiegati nel saggio sugli anelli, in particolare il concetto di stabilità. Si tratta di un articolo pubblicato nei Proceedings della Royal Society nel 1868, dal titolo lapidario: On governors. Allo scopo di imporre un determinato comportamento a un dato sistema, si può ricorrere a un disposi- 
tivo opportunamente progettato che, connesso al sistema stesso, ne garantisca il buon funzionamento. Nell'articolo citato, tale dispositivo è indicato come governor; oggi si usa piuttosto il termine controller in inglese, e controllore nella nostra lingua. L'insieme del sistema da governare e del suo controllore prende il nome di sistema di controllo. La scienza che si occupa dello studio di questi sistemi prende il nome di Automatica.

A titolo esemplificativo, soffermiamoci sul compito di imporre una certa temperatura in un locale. Nel periodo invernale, l'obiettivo è raggiunto mediante un dispositivo che regola la quantità di calore immessa nel locale, tipicamente tramite un termostato, che, dal confronto tra la temperatura attuale e temperatura desiderata, provvede ad accendere o spegnere la sorgente di calore. Un simile sistema prende più precisamente il nome di sistema di controllo retro-azionato poiché è dalla valutazione della differenza tra temperatura desiderata e temperatura effettiva che si decide l'intervento ottimale del termostato. La denominazione deriva dal fatto che la misurazione della temperatura effettuata nel locale deve essere riportata (portata indietro, retro-azionata) al termostato per il confronto con il riferimento di temperatura.

E' importante notare che i sistemi di controllo sono diffusissimi anche negli esseri viventi; in tal caso è la natura stessa ad aver impresso in un nostro organo la legge di controllo, senza che se ne abbia consapevolezza. Ad esempio, il complesso sistema di regolazione del glucosio nel sangue ha sede nel pancreas, un organo che produce due ormoni, l'insulina e il glucagone, aventi effetti contrastanti tra loro: il primo riduce la concentrazione di glucosio, mentre il secondo la aumenta. I due ormoni sono generati automaticamente in opportuna quantità a seconda che vi sia eccesso o carenza di glucosio, consentendo il mantenimento delle condizioni di benessere naturale dell'individuo. Il diabete è una malattia che insorge quando il pancreas non è più in grado di reagire al disallineamento tra concentrazione effettiva di glucosio e concentrazione ideale.

Per maggiori informazioni sull'Automatica si rimanda all'immensa letteratura disponibile, ad esempio al volume [5]; chi fosse interessato all'evoluzione storica della disciplina può fare riferimento a [6]-[10].

Ma torniamo all'articolo di Maxwell On Governors, dove l'autore prende in considerazione alcuni sistemi operanti in realtà fisiche diverse, in particolare il cosiddetto regolatore centrifugo. Questo 
dispositivo fu concepito per il controllo dei mulini a vento e poi adattato al problema del controllo della velocità dell'albero di macchine a vapore. Come noto, le macchine a vapore presero a svilupparsi all'inizio della rivoluzione industriale del 700, principalmente in Gran Bretagna, per la movimentazione dei treni. Erano però soggette a disturbi di varia natura, vuoi per le variazioni di carico vuoi per la variabilità delle caratteristiche del combustibile impiegato. Il regolatore centrifugo era un dispositivo consistente in una sorta di compasso ad apertura variabile il cui asse di rotazione era calettato sull'albero della macchina da regolare (Fig. 3). Incidentalmente, notiamo che questa immagine è diventata una sorta d'icona dell'Automatica, tanto da essere stata adottata come marchio da diversi istituti di ricerca nel settore e da essere riprodotta sulle copertine di molti libri. All'estremità del compasso erano collocate due sferette, cosicché, quando - ad esempio - la velocità di rotazione era eccessiva, la forza centrifuga agente sulle sferette portava ad un ampliamento dell'apertura del compasso; corrispondentemente, tramite un opportuno levismo, questa variazione si rifletteva sulla portata del vapore, e quindi sulla velocità di rotazione dell'albero, riportandola in modo automatico, senza intervento di un operatore umano, a valori accettabili.

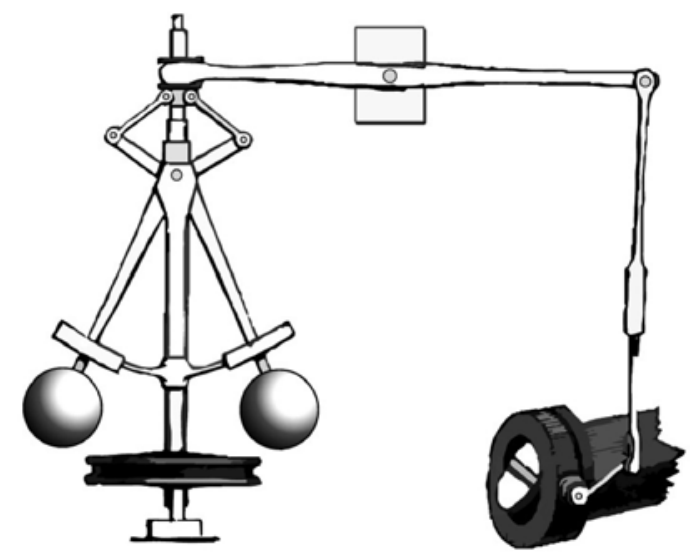

Fig. 3 - Il regolatore centrifugo.

Nel suo articolo del 1868, oltre al regolatore centrifugo, Maxwell tratta diversi altri sistemi di controllo, appartenenti a campi assai diversi. E' degno di nota osservare che tutti i dispositivi conside- 
rati sono descritti mediante sistemi dinamici lineari, seppur di diversa complessità. Ciò che li accomuna tutti quanti è che si tratta di sistemi retro-azionati. Corrispondentemente il concetto di controllore (governor) è comune:

"A governor is a part of a machine by means of which the velocity of the machine [...] is kept nearly uniform, not with standing variations in the driving power or the resistance. Most governors depend on the centrifugal force of a piece connected with a shaft of the machine. When the velocity increases, this force increases, and either increases the pressure of the piece against a surface or moves the piece, and so acts on a break or a valve".

Più avanti si legge:

"I propose [...] to direct the attention of engineers and mathematicians to the dynamical theory of such governors. It will be seen that the motion of a machine with its governor consists in general of a uniform motion, combined with a disturbance which may be expressed as the sum of several component motion. These components may be of four different kinds:

The disturbance may continually increase

It may continually diminish

It may be an oscillation of continually increasing amplitude

It may be an oscillating continually decreasing amplitude

The first and the third cases are evidently inconsistent with the stability of the motion, and the second and fourth alone are admissible in a good governor. This condition is mathematically equivalent to the condition that all the possible roots, and all the possible parts, of the impossible roots of a certain equation shall be negative. I have not been able completely to determine these conditions for equation of a higher degree than the third; but I hope that the subject will obtain the attention of mathematicians".

In ultima analisi, lo studio della stabilità porta al problema della risoluzione di un'equazione polinomiale a coefficienti reali, quella che oggi si chiama equazione caratteristica. Il risultato base è che un sistema è stabile se tutte le soluzioni della sua equazione caratteristica hanno parte reale negativa. Uno dei meriti di Maxwell fu di aver compreso che le cosiddette soluzioni impossibili, vale a dire le soluzioni complesse, sono altrettanto importanti delle soluzioni reali e anch'esse vanno analizzate nello studio della stabilità. Si noti poi che, secondo il particolare 
sistema considerato, l'equazione caratteristica ricavata dall'autore poteva avere grado diverso. E Maxwell termina riconoscendo di non essere stato in grado di ricavare una condizione di stabilità per polinomi di grado superiore a tre.

\section{STUDIO DELLE SOLUZIONI DI UNA EQUAZIONE ALGEBRICA}

Siamo dunque condotti ad un celebre problema, palestra di studio per molti scienziati: il problema della soluzione di equazioni algebriche di vario grado.

Come noto vi è una formula esplicita per le soluzioni delle equazioni di secondo grado. Ma anche le equazioni di terzo grado ammettono soluzione in forma chiusa. La storia della messa a punto di quest'ultima formula è davvero curiosa. La scoperta si deve a due matematici di grandissimo valore, il bresciano Niccolò Fontana, detto Tartaglia (1499-1557), e il bolognese Scipione del Ferro (1465-1526), che la ricavarono in modo indipendente l'uno dall'altro. Sia Tartaglia sia del Ferro, però, tennero la formula nel cassetto per parecchi anni. Tartaglia la rivelò in via del tutto confidenziale a Gerolamo Cardano (15011576). Quanto a del Ferro, confidò la soluzione a suo genero Annibale della Nave, che, a sua volta, la espose a Cardano durante una visita di quest'ultimo a Bologna. Fatto sta che la formula fu pubblicata nel 1545 nel libro di Cardano Ars Magna, universalmente considerato come il maggiore trattato scientifico del Rinascimento. Nel libro, Cardano ammette che la paternità della formula non è sua, riconoscendo che essa si deve a Tartaglia e a del Ferro. Tuttavia, Tartaglia se la prese a male, ed ebbe inizio una disputa che si protrasse per anni [11], [12]. Ricordiamo poi che Paolo Ruffini (1765-1822) provò che, dal quinto grado, non vi è una formula esplicita risolutiva.

In seguito a tutte queste considerazioni, sembrerebbe, dunque, che il problema dell'analisi di stabilità sia un problema arduo, soprattutto per sistemi complessi, di elevato ordine. A ben riflettere, però, l'analisi di stabilità non richiede la soluzione esplicita dell'equazione caratteristica, richiede molto meno: basta stabilire se tutte le soluzioni hanno parte reale negativa.

Maxwell cercò di ottenere una condizione necessaria e sufficiente, senza riuscirvi, come scrisse nel passaggio finale del suo articolo sopra citato. 


\section{IL PREMIO ADAMS DEL 1877 E IL CRITERIO DI ROUTH}

In seguito al suggerimento espresso da Maxwell nel finale del suo articolo del 1868, fu bandito un Premio Adams per la messa a punto di un criterio di stabilità. Il premio, assegnato nel 1877, andò a Edward J. Routh (1831-1907), anch'egli docente a Cambridge, per il suo saggio dal titolo $A$ treatise in the stability of a given state of motion. Routh aveva ricavato una condizione (necessaria e sufficiente) grazie alla quale era possibile stabilire se tutte lo soluzioni di una equazione algebrica avessero parte reale negativa, senza che fosse necessario ricavare le soluzioni dell'equazione stessa. La condizione richiedeva solamente la costruzione di una tabella i cui elementi si potevano ricavare con calcoli elementari dai coefficienti del polinomio di partenza. Nasceva così il celeberrimo Criterio di Routh, ${ }^{3}$ che è insegnato oggi in tutti i corsi di Automatica nel mondo, si veda ad esempio [5].

In realtà si può ben dire che Maxwell e Routh ebbero due destini incrociati. Nati entrambi nel 1831, furono compagni di studi a Cambridge. A quei tempi, era d'uso sottoporre gli allievi di quella Università ad una prova molto selettiva, una sorta di "competizione dei bravissimi”, denominata Mathematical Tripos, che si teneva alla fine del primo triennio di studi. I Tripos consistevano in un insieme di test matematici complessi, che potevano richiedere diversi giorni di lavoro da parte dei candidati. L'etimologia del termine Tripos non è chiara; potrebbe forse avere a che vedere con la parola tripode (tripod), lo sgabello a tre piedi che gli studenti usavano nei loro esami orali all'università. I primi classificati in questa competizione venivano inseriti in una lista nota come Wrangler list. Questo era un segno distintivo molto ambito, ed anche un prestigioso biglietto da visita per future occupazioni (non necessariamente universitarie, né unicamente di tipo scientifico, ad esempio per concorsi di giudice di tribunale). La classificazione ottenuta in queste prove era indicata con sigle sintetiche, ad esempio $7 \mathrm{~W}$ 1893 indica chi si classificò in settima posizione nei Tripos del 1893, mentre 12W 1905 sta per il dodicesimo classificato del 1905 (per la pre-

3 Nel 1894, Adolf Hurwitz (1859-1916), un matematico che lavorava al Politecnico di Zurigo (ETH), ricavò una condizione necessaria e sufficiente diversa ma equivalente a quella di Routh. Per questo il criterio viene anche denominato Criterio di Routh-Hurwitz. 
cisione, si tratta rispettivamente di Bertrand Russell e di Lord Keynes). Maxwell e Routh avevano partecipato entrambi ai Tripos del 1854, classificandosi ai primi due posti (1W 1854 e 2W 1854). Per l'esattezza, Routh era stato classificato al primo posto e Maxwell al secondo.

Una seconda analogia tra i due riguarda il premio Adams, che, come già detto, fu assegnato a Maxwell per il suo saggio sugli anelli di Saturno e, circa vent'anni dopo, a Routh per il suo studio sulla condizione generale di stabilità.

\section{EPILOGO}

Il saggio sugli anelli di Saturno rappresenta il debutto scientifico di James C. Maxwell, all'epoca studente universitario a Cambridge. Per la comprensione della costituzione degli anelli, si adotta un approccio molto originale, che anticipa la logica delle moderne tecniche d'identificazione. Se si considerano poi i fondamentali contributi successivi all'elettromagnetismo e ad altri campi, si comprende perché siano in molti a ritenere che Maxwell sia stato il maggiore scienziato dell'Ottocento. Sulla sua vita e sui suoi contributi, vi sono molte pubblicazioni, tra cui $[13]^{4}$ e [14]. Nella commemorazione per il centenario della sua nascita, Albert Einstein disse che Maxwell era stato "the most profound and the most fruitful scientist that physics has experienced since the time of Newton". Chi scrive si è chiesto spesso quali altri contributi l'illustre scienziato ci avrebbe potuto dare se un tumore non lo avesse sottratto alla vita a soli 48 anni.

4 Questo volume si compone di tre parti. La prima è articolata in 14 capitoli, i primi 13 dei quali sono dedicati alla biografia dello scienziato. I lavori di Maxwell sono raccolti nel capitolo 14 della prima parte, e nelle parti seconda e terza del volume. Per la precisione, gli scritti scientifici sono presentati nella parte seconda; il capitolo 14 della parte prima contiene invece tre scritti a carattere filosofico; infine la terza parte contiene le poesie di Maxwell. Queste ultime sono state tradotte in italiano con testo originale a fronte nel volume "Poesie" pubblicato da Archivio Dedalus Edizioni nel 2012. Il Capitolo 14 della Parte prima è stato tradotto in italiano e pubblicato con il titolo "Ultimi saggi di Cambridge" nel 2014, sempre da Archivio Dedalus Edizioni. E' ora in corso la traduzione in lingua italiana dei Cap. 1-13, la vera e propria biografia, che verrà pubblicata nel 2015, dal medesimo editore. L'Istituto Lombardo - Accademia di Scienze e Lettere ha in programma un evento nell'autunno 2015 per celebrare il completamento di questa poderosa opera. 


\section{BIBLIOGRAFIA}

[1] Simonyi K., A cultural bistory of Physics. CRC Press, Boca Raton, Florida, 2012.

[2] Bittanti S., Identificazione dei Modelli e Sistemi Adattativi. Pitagora Editrice, Bologna, prima edizione 1990, ultima edizione 2013.

[3] Lovett L., Horvath J., Cuzzi J., Saturn - a New View. Abrams, New York, 2006.

[4] Sassone Corsi E., Il Signore degli Anelli, Gremese, Roma, 2015.

[5] Bittanti S., Introduzione all'Automatica, Zanichelli, Bologna, 2014.

[6] Åström K.J., Kumar P.R., Control: A perspective, Automatica, 2014: vol. 50: 3-43.

[7] Bittanti S., Zibaldone di pensieri sull'evoluzione storica dell'Automatica, AEIT, 2014: 6: 30- 43. (numero speciale per il centenario della rivista AEIT).

[8] Bittanti S. (ed.), Control Science evolution. Proceedings of the Second Convegno Internazionale sui Problemi dell'Automatismo. Consiglio Nazionale delle Ricerche, Roma, 2008.

[9] Guardabassi G.O., The dawn of control science in Italy - from intuitive engineering to modern control theory. European Journal of Control: 2007: 13: 36-48.

[10] Lepschy A., Trent'anni di Automatica in Italia. Automazione e Strumentazione: 1997: 45: 91-97.

[11] Bittanti L., Di Nicolò Tartaglia, matematico bresciano. Apollonio, Brescia, 1871.

[12] Toscano F., La formula segreta, Sironi Editore, Milano, 2009.

[13] Campbell L., Garnett W., The life of James Clerk Maxwell. MacMillan and Co., London, 1882.

[14] James Clerk Maxwell, in: Alessandro Volta, le onoranze del 1878 all'Università di Pavia, a cura di Cantoni V., Morando P.A., 132-139, Silvana Editoriale, Cinisello Balsamo, 2011. 